\title{
IDENTIFICACIÓN DE TRANSTORNOS RELACIONADOS CON EL USO DE ALCOHOL EN ALUMNOS INGRESANTES A LA UNIVERSIDAD NACIONAL JORGE BASADRE GROHMANN TACNA AÑO 2005
}

Responsable: Mgr. Rina Álvarez Becerra

\begin{abstract}
RESUMEN
El problema de investigación se centra en la identificación de transtornos relacionados con el uso de alcohol . El objeto de estudio son los ingresantes 2005 a la Universidad Nacional Jorge Basadre Grohamnn de Tacna.

El diseño de investigación es no experimental, transversal - descriptivo. La muestra se ha calculado con un $95 \%$ de confianza y un $5 \%$ de error, el tipo de muestreo ha sido el aleatorio simple con afijación proporcional. Se ha utilizado como instrumento de recolección de datos el cuestionario AUDIT semiestructurado que consta de 10 item y que se puntúan en una escala del 0 al 32 .

Se ha concluido que la mayoría de los ingresantes, sin distinción de género, no presenta transtornos relacionados con el uso de alcohol, mientras que una minoría sí los presenta con una puntuación que se sitúa por debajo del punto de corte : 8 , lo que significa un bajo riesgo. Los hallazgos empíricos no confirman la hipótesis de investigación.
\end{abstract}

\section{ABSTRACT}

The research is centered on the identification of disorders related to the alcohol usage. The objective of the investigation is the population who got a vacancy to the National University Jorge Basadre Grohmann. of Tacna in 2005.

The design of the investigation is no experimental, transversal descriptive. The sample has been calculated with a $95 \%$ of confidence and $5 \%$ of error, the kind of this sample has been the simple aleatory with proportional detached. The semi-structured questionaire AUDIT which has 10 items punctuated from 0 to 32 has been used as an instrument to collect data.

As a conclusion; most of the students of the population without considering gender, does not present disorders related to the alcohol usage, while the minority of them presents this trouble with a punctuation under the cut point :8 which means a low risk. The empiric findings do not confirm the hypothesis of the investigation.

\section{INTRODUCCIÓN}

La presente investigación plantea el problema de los transtornos relacionados con el uso de alcohol en jóvenes universitarios, pues las investigaciones al respecto revelan que los estilos de vida de éstos han cambiado y el consumo de alcohol en este grupo etáreo se ha distanciado de los patrones tradicionales para convertirse en un elemento articulador de la vida social entre pares, lo que muchas veces les ocasiona problemas académicos en el ámbito universitario, problemas sociofamiliares y de salud, lo que deteriora su calidad de vida y limita las posibilidades de éxito.

El propósito de este estudio es la identificación temprana de los problemas de abuso de bebidas alcohólicas en los ingresantes 2005 a la Universidad Nacional Jorge Basadre Grohmann de ambos sexos, que no presentan aún una dependencia física o que aún no están afectados por problemas crónicos, físicos o psicosociales.

El alcoholismo ha pasado a ser definido recientemente, y quizá de forma más acertada, como una enfermedad compleja en sí, con todas sus consecuencias . Inclusive en el año 1977 la OMS propuso que se dejara de hablar de alcoholismo para hacerlo de "Problemas relacionados con el alcohol", que en el caso de los jóvenes universitarios el consumo del alcohol muchas veces perturba, por un lado, su normal desenvolvimiento académico en términos de bajo rendimiento académico, deserción y ausentismo, $y$, por otro lado, las relaciones sociales con sus pares y familia.

Los antecedentes evidencian que en EEUU, anualmente 85000 personas fallecen de forma directa por abuso de alcohol, y a esta cifra hay que sumar la de los accidentes y la discapacidad o comorbilidad generada por esta toxicomanía. En México, el $60 \%$ de ingresantes del nivel medio y superior están en riesgo por el consumo de alcohol y $50 \%$ por el consumo de tabaco.

España tiene más de 1265000 alcohólicos mayores de 16 años, pero lo sintomático del 
futuro es que tiene más de 800000 menores de entre 12 y 15 años que ya consumen alcohol regularmente. E.Freyre refiere que en el Perú las bebidas fermentadas y destiladas más usadas en la zona urbana son la cerveza, vino y pisco y cañazo respectivamente; en la zona andina y en partes rurales de la costa la chicha de jora y en la selva el masato de yuca.

\section{MATERIAL Y MÉTODOS}

La población de base objeto de estudio la componen 1440 ingresantes el año 2005 a las treinta Escuelas Académicas de las quince Facultades que tiene la UNJBG.

El tamaño de muestra se definió considerando un nivel de confianza del $95 \%$ ( $Z=$ 1,96 ) y un error del $5 \%$, la que quedó conformada por 304 estudiantes. Para la selección de la muestra se utilizó el método de muestreo estratificado con afijación proporcional por Facultades.

El instrumento utilizado fue el cuestionario AUDIT test de identificación de transtornos Una relacionados con el uso de alcohol. Es una encuesta semiestructurada que consta de 10 preguntas, que se puntúan según la frecuencia de 0 (Nunca) a 4 (Diariamente) en las ocho primeras preguntas y de 0 a 2 en las dos últimas, la puntuación de la escala oscila de 0 a 36 . Una puntuación de 8 ó más revela la existencia de problemas relacionados con el alcohol; una puntuación superior a 20 es ya señal de dependencia.

\section{RESULTADOS}

\section{Variable Ingesta de Alcohol:}

Del Gráfico 1 podemos determinar que en el grupo de ingresantes de sexo femenino y masculino, el $55,4 \%$ y 37,4 respectivamente informan que nunca consumen bebidas alcohólicas. Sin embargo, un porcentaje nada despreciable de $36,3 \%$ y 51,0 para mujeres y varones respectivamente revela que la frecuencia con que consume bebidas alcohólicas es de una vez al mes o menos.

GRÁFICO 1. Frecuencia de ingesta de Alcohol en Ingresantes según sexo Universidad Nacional Jorge Basadre Grohmann 2005.

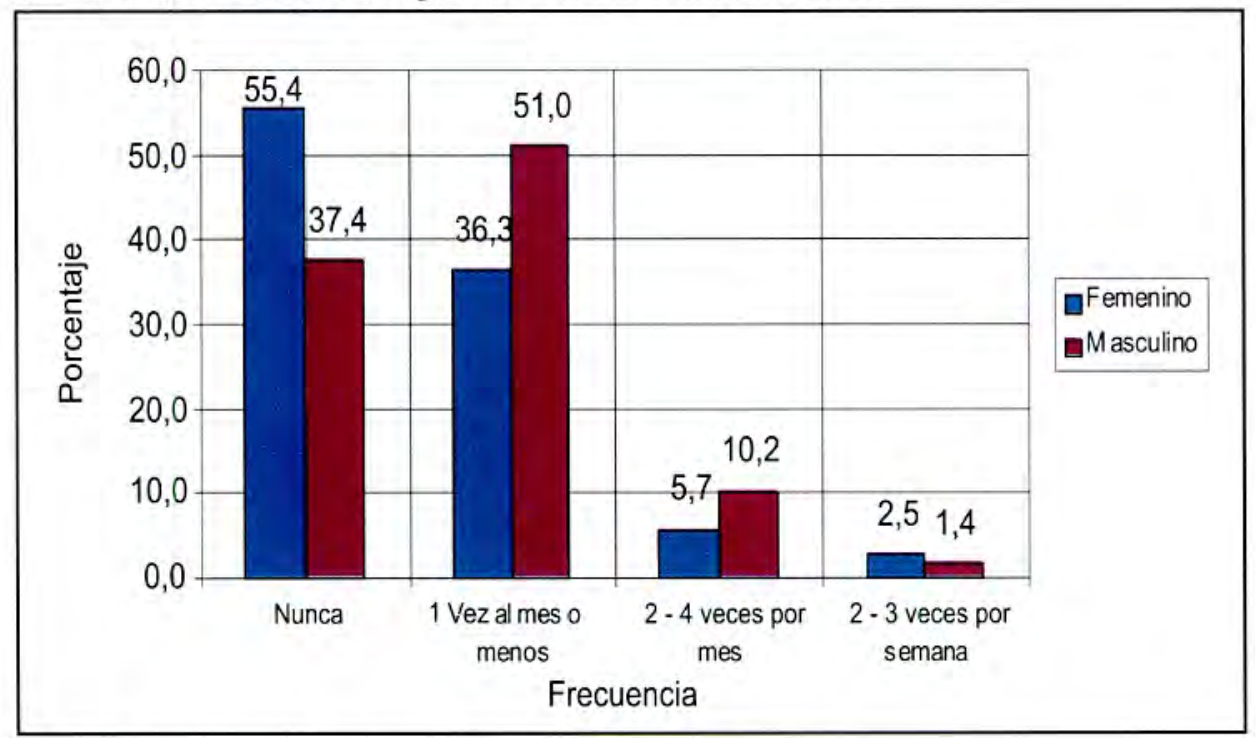

Fuente: Cuestionario $\mathrm{N}^{\circ} 05$

\section{Variable Dependencia:}

El Gráfico 2 exponen los resultados para la variable Incumplimiento de obligaciones a causa del alcohol. Los porcentajes obtenidos revelan que del total de ingresantes de sexo femenino encuestados $94,9 \%$ (147) afirma que nunca durante el último año ha dejado de cumplir con sus obligaciones a causa del alcohol, a diferencia del grupo de varones con un $90,5 \%$ (133), lo que se puede interpretar que la gran mayoria no abandona sus aficiones a causa de las bebidas alcohólicas.

En contraste observamos un porcentaje de $4,5 \%(7)$ y $5,4 \%$ ( 8 ) para el grupo de mujeres y varones respectivamente, que afirman que menos de una vez al mes han dejado de cumplir con sus obligaciones a causa del alcohol, lo que implicaria la dependencia al alcohol en términos de abandonar sus obligaciones escolares 0 de trabajo, según el caso. 
GRÁFICO 2. Incumplimiento de Responsabilidades debido al Consumo de Alcohol en Ingresantes Según Sexo Universidad Nacional Jorge Basadre Grohmann 2005.

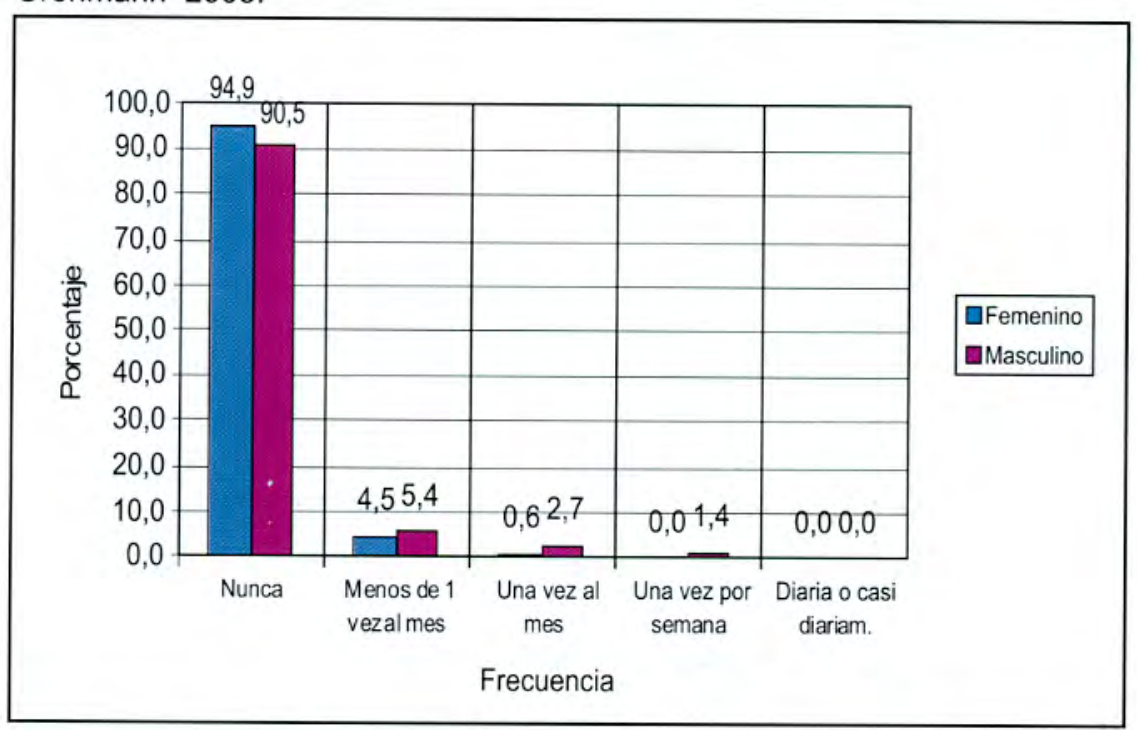

Fuente: Cuestionario $\mathrm{N}^{\circ} 09$.

\section{Variable Reacciones Negativas a la bebida:}

GRÁFICO 3. Incapacidad de recordar sucesos ocurridos en el lapso en que se consume alcohol según sexo Universidad Nacional Jorge Basadre Grohmann 2005.

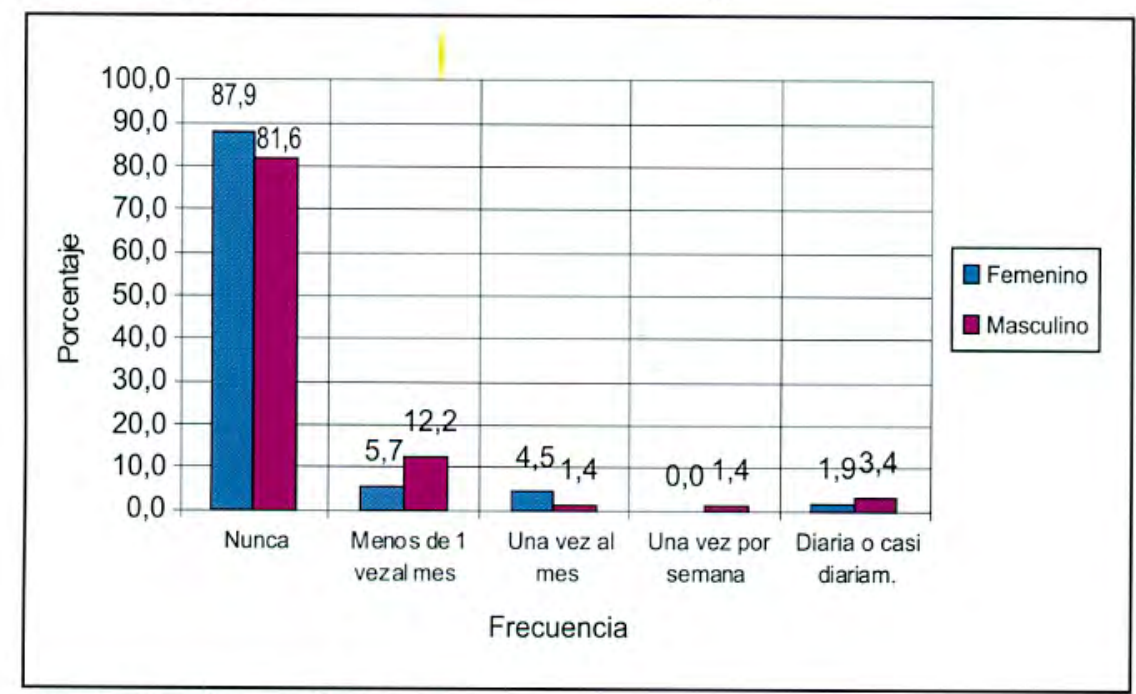

Fuente: Cuestionario $\mathrm{N}^{\circ} 12$.

El Gráfico 3 revela información acerca de las reacciones negativas a la bebida como es la "Incapacidad de recordar sucesos ocurridos en el lapso en que se consume alcohol"; encontrándose que cuando se les pregunta a los encuestados "¿Con cuánta frecuencia durante el último año ha sido incapaz de recordar lo que había pasado la noche anterior porque habia estado bebiendo?", un $87,9 \%$ de las ingresantes de sexo femenino y un $81,6 \%$ de sexo masculino respondieron que "Nunca".

Para la categoría de respuesta de menos de una vez al mes, los porcentajes difieren según el género, ya que las mujeres presentan un porcentaje de $5,7 \%$ y los varones de $12,2 \%$, interpretándose que éstos últimos presentan mayor frecuencia, el doble, de recordar lo que habia pasado la noche anterior después de haber bebido. Las respuestas se refieren a los sucesos ocurridos en el último año.

Finalmente, la frecuencia diariamente o casi diariamente con que se dio el último año la incapacidad de recordar lo sucedido la noche anterior por haber estado bebiendo, en el caso de las ingresantes de sexo femenino es de $1,9 \%$ y para los de sexo masculino de $3,4 \%$, casi el doble 
en comparación con el valor que presentan las damas.

\section{Variable Problemas Derivados del consumo de Alcohol:}

En el Gráfico 4 se aprecia que en relación con la categoría de respuesta de no haber tenido accidentes ni haber herido a alguien como consecuencia del consumo de alcohol, el grupo de mujeres presenta un valor porcentual de $98,7 \%$ y el de varones un 95,2 .

GRÁFICO 4. Ocurrencia de accidentes debido al consumo de alcohol en ingresantes según sexo. Universidad Nacional Jorge Basadre Grohmann 2005.

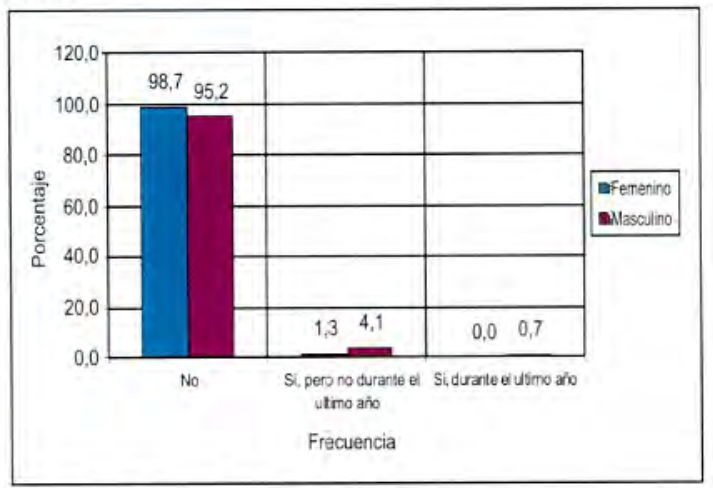

Fuente: Cuestionario $N^{0} 13$.

\section{CONCLUSIONES}

1. En relación con la variable de estudio TRANSTORNOS RELACIONADOS CON EL USO DE ALCOHOL, la valoración se ha hecho en relación a la puntuación AUDIT en una escala de 0 a 32, comprende la sumatoria de las puntuaciones de las variables intermedias: ingesta de alcohol, dependencia del alcohol, reacciones negativas a la bebida y problemas derivados del consumo de alcohol. En consecuencia, las conclusiones aluden a los resultados en relación con la puntuación total.

2. El $12,2 \%$, sin distinguir género, presenta transtornos relacionados con el uso de alcohol, y la puntuación en la escala AUDIT de 0 a 32 se ubica en 3,2. La media es de 2,81 , con un intervalo de confianza al $95 \%$ de 2,40 a 3,23 . El $50 \%$ de los valores tiene una puntuación de 0 a 4 , la misma que no alcanza el punto de corte que es de 8 ; lo que significa que la probabilidad de riesgo es baja.

3. Los hallazgos empíricos no permiten confirmar la hipótesis de investigación, que afirma que una proporción superior al $50 \%$ presenta transtornos relacionados con el uso del alcohol, ya que el $87,8 \%$ de la población muestral, sin distinguir género, que no presenta transtornos relacionados con el uso de alcohol (puntuación 0 ); y el porcentaje de $12,2 \%$ que presenta transtornos no supera la puntuación 8, considerada en la escala de AUDIT como punto de corte para la identificación temprana de problemas de abuso de bebidas alcohólicas.

4. El estadístico de prueba seleccionado fue la Prueba de Hipótesis para una Proporción; se eligió un nivel de significación de 0,05 . Se procedió al cálculo y se obtuvo un valor de - 14,89 que pertenece a la región de aceptación; , entonces no se rechaza la hipótesis nula ( $\mathrm{Ho}: \mathrm{p}=$ po ) y se concluye al nivel del $5 \%$ de significancia que la proporción de ingresantes que padecen transtornos relacionados con el uso del alcohol es menor del $50 \%$.

\section{BIBLIOGRAFÍA}

Freyre Román, Eleodoro ( 1994) La Salud del adolescente. aspectos médicos y psicosociales. Edit. Consejo Nacional de Ciencia y Tecnologia, Lima.

Morris, Charles G. ( 1992) Psicología. Un nuevo Enfoque. Tr.del inglés por Guillermina Cuevas Mesa, $7 \mathrm{ma}$ ed. Edit. Prentice - Hall Hispanoamericana, S. A. México, 687 pp.

Toledo Mayo, Luis (1984) Drogas. Uso y Abuso. 5ta ed., Edit.Consejo Nacional de Ciencia y Tecnología, Lima.

Manzarbeitia, Jorge, "Análisis. Consecuencias del abuso del consumo de alcohol". Revista Española de Economía de la Salud. http:// economía de la salud.com / Ediciones/42/08/pdf/epidemiologia.pdf. Consulta: 13.03.06. 